\title{
Global Attractors for Multivalued Random Dynamical Systems*
}

\author{
T. Caraballo ${ }^{1}$, J.A. Langa ${ }^{1}$ and J. Valero ${ }^{2}$ \\ ${ }^{1}$ Departamento de Ecuaciones Diferenciales y Análisis Numérico, Universidad de Sevilla, \\ Apdo. de Correos 1160, 41080-Sevilla, Spain. \\ e-mails: caraball@numer.us.es ; langa@numer.us.es \\ 2 CEU San Pablo-Elche, Comisario 3, 03203 Elche, Alicante. Spain. \\ e-mail: ceuade@ctv.es
}

\begin{abstract}
We introduce the concept of multivalued random dynamical system (MRDS) as a measurable multivalued flow satisfying the cocycle property. We show how this is a suitable framework for the study of the asymptotic behaviour of some multivalued stochastic parabolic equations by generalizing the concept of global random attractor to the case of a MRDS.
\end{abstract}

Keywords: global attractor, multivalued random dynamical systems, stochastic differential inclusions.

AMS 1991 Subject Classification: 58F39, 35B40, 35K55, 35K22, 35R60, 60H15.

\section{Introduction}

The study of the qualitative behaviour of ordinary and partial differential equations is one of the most developed branches in this field. When a phenomenon from Physics, Chemistry, Biology, Economics can be described by a system of differential equations

*The research of the two first authors has been partially supported by Proyecto DGICYT No. PB981134. The third author has been supported by PB-2-FS-97 grant (Fundación Séneca de la Comunidad Autónoma de Murcia). 
where the existence of global solutions can be assured, one of the most interesting problems is to know what is the asymptotic behaviour of the system when time grows to infinite. The study of the asymptotic behaviour of the system is giving us relevant information about "the future" of the phenomenon described in the model. In this context, the concept of global attractor has become a very useful tool to describe the long-time behaviour of many important differential equations (see, among others, Ladyzhenkaya [18], Babin and Vishik [5], Hale [17], Temam [23]). Most of this theory has been successfully and deeply developed for autonomous deterministic partial differential equations.

However, some difficulties appear when we have to work without uniqueness of solutions in the system or when the model is better described by, for instance, a differential inclusion. In these cases, it has been shown that the theory of multivalued flows makes suitable the treatment of the asymptotic behaviour of these differential equations and inclusions (Ball [6], Melnik and Valero [19]). It is proved in these works that we can generalize the classical dynamical system theory to these more general cases.

A new and different difficulty appears when we a random term is added to the deterministic equation, a white noise for instance, so that the corresponding stochastic partial differential equation must be treated in a different way. Firstly, the equation becomes nonautonomous, which makes necessary the introduction of a two time dependent process instead of a semigroup. Moreover, the strong dependence on the random term adds another difficulty. The new and rapidly growing theory of random dynamical systems (Arnold [2]) has become the appropriate tool for the study of many important random and stochastic equations. In this framework, Crauel and Flandoli [11] (see also Schmalfuss [22]) introduced the concept of random attractor as a proper generalization (see Caraballo et al.[9]) of the corresponding (determininstic) global attractor.

The joint treatment of multivalued functions and stochastic terms in a differential equation makes difficult even the existence and uniqueness of solutions for these systems (Ahmed [1], Da Prato and Frankowska [14], among others). In this paper we study the asymptotic behaviour of some stochastic inclusions by introducing previously the corresponding concept of random attractor for this case.

The paper is divided into two parts. In the first one (Section 2), we generalize the concept of random dynamical system in Arnold [2] to the case of multivalued functions. A multivalued random dynamical system (MRDS) will be a multivalued measurable map satisfying the cocycle property. In this framework, we can introduce the concepts of invariance, absorbtion and attraction which will lead us to a general result of existence and uniqueness of random attractors (in the sense of Crauel and Flandoli [11]) for MRDS.

In the second part (Section 3), we apply the previous results to some stochastic differential inclusions. Special care must be paid to the correct definition of the corresponding multivalued stochastic flow, as for example in proving the measurability condition or the cocycle property. Next, it is shown that conditions for the existence of random attractors are satisfied. Some particular examples are also given in Section 4. 
Finally, some remarks and conclusions appear in the last section.

\section{Multivalued random dynamical systems and at- tractors}

\subsection{Multivalued random dynamical systems}

Let $\left(X, d_{X}\right)$ be a complete and separable metric space with the Borel $\sigma$-algebra $\mathcal{B}(X)$. Let $(\Omega, \mathcal{F}, \mathbb{P})$ be a probability space and $\theta_{t}: \Omega \rightarrow \Omega$ a measure preserving group of transformations in $\Omega$ such that the map $(t, \omega) \mapsto \theta_{t} \omega$ is measurable and satisfying

$$
\theta_{t+s}=\theta_{t} \circ \theta_{s}=\theta_{s} \circ \theta_{t} ; \quad \theta_{0}=I d
$$

The parameter $t$ takes values in $\mathbb{R}$ endowed with the Borel $\sigma$-algebra $\mathcal{B}(\mathbb{R})$.

Definition 1 A set valued map $G: \mathbb{R}^{+} \times \Omega \times X \rightarrow C(X)(C(X)$ denotes the set of nonempty closed subsets of $X)$ is called a multivalued random dynamical system (MRDS) if is measurable (see Aubin and Frankowska [4], definition 8.1.1) and satisfies

i) $G(0, \omega)=I d$ on $X$;

ii) $G(t+s, \omega) x=G\left(t, \theta_{s} \omega\right) G(s, \omega) x \quad$ (cocycle property) for all $t, s \in \mathbb{R}^{+}, x \in X, \omega \in \Omega$.

Remark 1 When ii) holds identically, we call $G$ a perfect cocycle. We call $G$ a crude cocycle if ii) holds for fixed $s$ and all $t \in \mathbb{R}^{+}, x \in X, \mathbb{P}$-a.s. (where the exceptional set $N_{s}$ can depend on $s)$. We call $G$ a very crude cocycle if ii) holds for fixed $s, t \in \mathbb{R}^{+}$, for all $x \in X, \mathbb{P}$-a.s. (where the exceptional set $N_{s, t}$ can depend on both $s$ and $t$ ). However, it is worth pointing out that in the applications of the following section we shall obtain a map satisfying ii) identically.

Remark 2 Throughout this paper all assertions about $\omega$ are assumed to hold on a $\theta_{t}$ invariant set of full measure (unless otherwise stated).

Recall the definition of Hausdorff semi-distance between bounded sets of $X$. For any $A, B \subset X$ bounded put $\operatorname{dist}(A, B)=\operatorname{supinf}_{y \in A} d_{X}(y, x)$.

Definition 2 The MRDS $G$ is said to be upper semicontinuous if for all $t \in \mathbb{R}^{+}$and $\omega \in \Omega$ it follows that given $x \in X$ and a neighbourhood of $G(t, \omega) x, \mathcal{O}(G(t, \omega) x)$, there exists $\delta>0$ such that if $d_{X}(x, y)<\delta$ then

$$
G(t, \omega) y \subset \mathcal{O}(G(t, \omega) x) .
$$


On the other hand, $G$ is called lower semicontinuous if for all $t \in \mathbb{R}^{+}$and $\omega \in \Omega$, given $x_{n} \rightarrow x(n \rightarrow+\infty)$ and $y \in G(t, \omega) x$, there exists $y_{n} \in G(t, \omega) x_{n}$ such that $y_{n} \rightarrow y$.

It is said to be continuous if it is upper and lower semicontinuous.

Remark 3 Note that these two definitions are not equivalent in general, as can be seen in easy examples (Aubin and Frankowska [4], Section 1.4).

Remark 4 Note that if a map $G(t, \omega)$ is upper semicontinuous at $x_{0}$, then $\forall \varepsilon>0$ there exists $\delta(\varepsilon)>0$ such that

$$
\operatorname{dist}\left(G(t, \omega) y, G(t, \omega) x_{0}\right) \leq \varepsilon,
$$

for any $y$ satisfying $d_{X}\left(y, x_{0}\right) \leq \delta(\varepsilon)$. The converse is true when $G(t, \omega) x_{0}$ is compact.

On the other hand, if $\forall \varepsilon>0$ there exists $\delta(\varepsilon)>0$ such that

$$
\operatorname{dist}\left(G(t, \omega) x_{0}, G(t, \omega) y\right) \leq \varepsilon,
$$

for any $y$ satisfying $d_{X}\left(y, x_{0}\right) \leq \delta(\varepsilon)$, then $G(t, \omega)$ is lower semicontinuous at $x_{0}$. The converse is true when $G(t, \omega) x_{0}$ is compact (see Aubin and Cellina [3, p.66], Propositions 2 and 3).

\subsection{Existence of global random attractors}

In this section we generalize the concept of random attractor to the case of a MRDS and prove a general result for the existence and uniqueness of attractors. Firstly we need some definitions.

Definition 3 A closed random set $D$ is a map $D: \Omega \rightarrow C(X)$, which is measurable. The measurability must be understood in the sense of Castaing and Valadier [10] for measurable multifunctions, that is, $\{D(\omega)\}_{\omega \in \Omega}$ is measurable if given $x \in X$ the map

$$
\omega \in \Omega \mapsto \operatorname{dist}(x, D(\omega))
$$

is measurable.

A closed random set $D(\omega)$ is said to be negatively (resp. strictly) invariant for the $\operatorname{MRDS} G$ if

$$
D\left(\theta_{t} \omega\right) \subset G(t, \omega) D(\omega)\left(\text { resp. } D\left(\theta_{t} \omega\right)=G(t, \omega) D(\omega)\right), \quad \forall t \in \mathbb{R}^{+}, \omega \in \Omega .
$$

Let us assume the following conditions for the MRDS $G$ :

(H1) There exists an absorbing random compact set $B(\omega)$, that is, for $\mathbb{P}$-almost all $\omega \in \Omega$ and every bounded set $D \subset X$, there exists $t_{D}(\omega)$ such that for all $t \geq t_{D}(\omega)$

$$
G\left(t, \theta_{-t} \omega\right) D \subset B(\omega)
$$


(H2) $G(t, \omega): X \rightarrow C(X)$ is upper semicontinuous, for all $t \in \mathbb{R}^{+}$and $\omega \in \Omega$.

Remark 5 We note that, although the measurability of the set $B(\omega)$ is not needed, in the applications considered in this paper this property is satisfied.

Define the limit set $\Lambda(D, \omega)=\Lambda_{D}(\omega)$ of a bounded set $D \subset X$ as

$$
\Lambda_{D}(\omega)=\cap_{T \geq 0} \overline{\cup_{t \geq T} G\left(t, \theta_{-t} \omega\right) D} .
$$

Lemma $1 \Lambda_{D}(\omega)$ is the set of limits of all converging sequences $\left\{x_{n}\right\}_{n \geq 1}$, where $x_{n} \in$ $G\left(t_{n}, \theta_{-t_{n}} \omega\right) D, t_{n} \nearrow+\infty$.

Proof. The proof follows the same lines as Lemma 1 in Melnik and Valero [19].

We can now prove the following proposition:

Proposition 2 Assume conditions (H1) and (H2) hold. Then, for $\mathbb{P}$-almost all $\omega \in \Omega$ and every $D \subset X$ bounded, it follows:

i) $\Lambda_{D}(\omega) \subset B(\omega)$ is nonvoid and compact.

ii) $\Lambda_{D}(\omega)$ is negatively invariant, that is, $G(t, \omega) \Lambda_{D}(\omega) \supseteq \Lambda_{D}\left(\theta_{t} \omega\right)$ for all $t \in \mathbb{R}^{+}$. If $G$ is lower semicontinuous, then $\Lambda_{D}(\omega)$ is strictly invariant.

iii) $\Lambda_{D}(\omega)$ attracts $D$,

$$
\lim _{t \rightarrow+\infty} \operatorname{dist}\left(G\left(t, \theta_{-t} \omega\right) D, \Lambda_{D}(\omega)\right)=0 .
$$

Proof.

i) Since for $\mathbb{P}$-almost all $\omega \in \Omega$ and any bounded $D \subset X$ we can write

$$
\Lambda_{D}(\omega)=\cap_{T \geq T_{D}(\omega)} \overline{\cup_{t \geq T} G\left(t, \theta_{-t} \omega\right) D} \subset B(\omega),
$$

we obtain immediately that $\Lambda_{D}(\omega)$ is compact.

Now let $d_{n} \in G\left(t_{n}, \theta_{-t_{n}} \omega\right) D, t_{n} \nearrow+\infty$. Since $B(\omega)$ is compact, there exists a converging subsequence $d_{n_{j}}$ of $d_{n}$. Therefore, in view of Lemma $1, d=\lim _{n \rightarrow+\infty} d_{n_{j}} \in$ $\Lambda_{D}(\omega)$, which proves that $\Lambda_{D}(\omega)$ is non-empty.

ii) We will firstly see that

$$
\Lambda_{D}\left(\theta_{t} \omega\right) \subset G(t, \omega) \Lambda_{D}(\omega), \quad t \geq 0 .
$$

Given $y \in \Lambda_{D}\left(\theta_{t} \omega\right)$, there exist $t_{n} \nearrow+\infty, y_{n} \in G\left(t_{n}, \theta_{-t_{n}} \theta_{t} \omega\right) D$ such that $y=$ $\lim _{n \rightarrow \infty} y_{n}$. On the other hand,

$$
\begin{aligned}
G\left(t_{n}, \theta_{-t_{n}} \theta_{t} \omega\right) D & =G\left(t+t_{n}-t, \theta_{-t_{n}} \theta_{t} \omega\right) D \\
& =G(t, \omega) G\left(t_{n}-t, \theta_{-\left(t_{n}-t\right)} \omega\right) D .
\end{aligned}
$$


Thus, there exists $x_{n} \in G\left(t_{n}-t, \theta_{-\left(t_{n}-t\right)} \omega\right) D$ such that $y_{n} \in G(t, \omega) x_{n}$. As $t_{n}-$ $t \nearrow+\infty$, by $(H 1)$ there exists $x_{n_{j}} \rightarrow x_{0} \in \Lambda_{D}(\omega) \cap B(\omega)$. Since $G(t, \omega)$ is upper semicontinuous with closed values, it has a closed graph (see Aubin and Frankowska [4], Proposition 1.4.8.), so that

$$
y \in G(t, \omega) x_{0} \subset G(t, \omega) \Lambda_{D}(\omega) .
$$

Let us prove the other inclusion. Suppose now that $G(t, \omega)$ is lower semicontinuous and take $z \in G(t, \omega) \Lambda_{D}(\omega)$. Then $z \in G(t, \omega) y$, with $y=\lim _{n \rightarrow+\infty} y_{n}, y_{n} \in$ $G\left(t_{n}, \theta_{-t_{n}} \omega\right) D$, so that

$$
z \in G(t, \omega)\left(\lim _{n \rightarrow+\infty} y_{n}\right) .
$$

By the lower semicontinuity we get that there exists $z_{n} \in G(t, \omega) y_{n}$ such that $z_{n} \rightarrow z$. By the cocycle property

$$
z_{n} \in G(t, \omega) y_{n} \subset G\left(t+t_{n}, \theta_{-t_{n}} \omega\right) D,
$$

so that $z \in \Lambda_{D}\left(\theta_{t} \omega\right)$.

iii) Finally, we prove that $\Lambda_{D}(\omega)$ attracts $D$. If not, there exist $\delta>0, t_{n} \nearrow+\infty, d_{n} \in D$, $y_{n} \in G\left(t_{n}, \theta_{-t_{n}} \omega\right) d_{n}$, such that

$$
\operatorname{dist}\left(y_{n}, \Lambda_{D}(\omega)\right) \geq \delta
$$

But, by (H1), for $n$ large enough $G\left(t_{n}, \theta_{-t_{n}} \omega\right) d_{n} \subset B(\omega)$, so that there exist converging subsequences of any sequence in $\left\{G\left(t_{n}, \theta_{-t_{n}} \omega\right) d_{n}\right\}_{n \geq 0}$. If we denote by $y$ one of these limits we get that $y \in \Lambda_{D}(\omega)$, which is a compact set, so that we get a contradiction.

Definition 4 The closed random set $\omega \mapsto \mathcal{A}(\omega)$ is called a global random attractor of the $M R D S G$ if $\mathbb{P}-$ a.s.

i) $G(t, \omega) \mathcal{A}(\omega)=\mathcal{A}\left(\theta_{t} \omega\right)$, for all $t \geq 0$, (that is, it is strictly invariant);

ii) for all bounded $D \subset X$,

$$
\lim _{t \rightarrow+\infty} \operatorname{dist}\left(G\left(t, \theta_{-t} \omega\right) D, \mathcal{A}(\omega)\right)=0
$$

iii) $\mathcal{A}(\omega)$ is compact.

We can now prove the following theorem on the existence of random attractors for MRDS: 
Theorem 3 Let $(H 1)-(H 2)$ hold, the map $(t, \omega) \in \mathbb{R}^{+} \times \Omega \mapsto \overline{G(t, \omega) D}$ be measurable for all deterministic bounded sets $D \subset X$, and the map $x \in X \mapsto G(t, \omega) x$ have compact values. Then,

$$
\mathcal{A}(\omega)=\overline{\cup_{D \subset X_{\text {bounded }}} \Lambda_{D}(\omega)}
$$

is a global random attractor for $G$ (measurable with respect to $\mathcal{F}$ ). It is unique and the minimal closed attracting set.

\section{Proof.}

Properties ii)-iii) from Definition 6 are obvious from the previous proposition.

For i), we note that by Proposition 2

$$
\begin{aligned}
\mathcal{A}\left(\theta_{t} \omega\right) & =\overline{\cup_{D \subset X} \Lambda_{D}\left(\theta_{t} \omega\right)} \subset \overline{\cup_{D \subset X} G(t, \omega) \Lambda_{D}(\omega)} \subset \overline{G(t, \omega) \cup_{D \subset X} \Lambda_{D}(\omega)} \\
& \subset G(t, \omega) \overline{\cup_{D \subset X} \Lambda_{D}(\omega)}=G(t, \omega) \mathcal{A}(\omega) .
\end{aligned}
$$

The last inclusion is a consequence of having $G(t, \omega)$ a closed graph. Indeed, write

$$
F=\cup_{D \subset X} \Lambda_{D}(\omega)
$$

and take $y \in \overline{G(t, \omega) F}$. Then, there exists $y_{n} \in G(t, \omega) z_{n}, z_{n} \in F$ with $y_{n} \rightarrow y$. Since $\mathcal{A}(\omega)$ is compact $\mathbb{P}$-a.s., there exists $z \in \mathcal{A}(\omega)$ such that $z_{n} \rightarrow z$ (accurate to a subsequence). Since $G(t, \omega)$ has closed graph, $y \in G(t, \omega) z$.

For the uniqueness, we follow the same lines as in Crauel [13]: let $\mathcal{E}(\omega)$ be a negatively invariant closed random set and for $D \subset X$ bounded define $F=\{\omega \in \Omega: \mathcal{E}(\omega) \subset D\}$. If we write

$$
F_{\infty}=\cap_{N \in \mathbb{N}} \cup_{n \geq N} \theta_{n} F=\left\{\omega: \theta_{-n} \omega \in F \text { infinitely often }\right\},
$$

we have that, by Poincare's recurrence theorem applied to the pair $\left(\mathbb{P}, \theta_{n}\left(=\theta_{1}^{n}\right)\right)$,

$$
\mathbb{P}\left(F_{\infty}\right) \geq \mathbb{P}(F)
$$

Now, if $\omega \in F_{\infty}, \mathcal{E}\left(\theta_{-n} \omega\right) \subset D$ for infinitely many $n \in \mathbb{N}$, so that, by the invariance of $\mathcal{E}(\omega)$

$$
\mathcal{E}(\omega) \subset G\left(n, \theta_{-n} \omega\right) \mathcal{E}\left(\theta_{-n} \omega\right) \subset G\left(n, \theta_{-n} \omega\right) D \text { for infinitely many } n \in \mathbb{N} \text {. }
$$

Consequently, $\mathcal{E}(\omega) \subset \cup_{n \geq \mathbb{N}} G\left(n, \theta_{-n} \omega\right) D$ for all $N \in \mathbb{N}$, and so, by definition of the omega limit set of $D$,

$$
\mathcal{E}(\omega) \subset \Lambda_{D}(\omega)
$$

We have shown that

$$
\mathbb{P}\left(\mathcal{E}(\omega) \subset \Lambda_{D}(\omega)\right) \geq \mathbb{P}(\mathcal{E}(\omega) \subset D)
$$


On the other hand, by Proposition 3.15 in Crauel [12], if $\mathcal{E}(\omega)$ is a compact random set, for $\varepsilon>0$ there exists $K_{\varepsilon} \subset X$ compact such that

$$
\mathbb{P}\left(\mathcal{E}(\omega) \subset K_{\varepsilon}\right)>1-\varepsilon,
$$

and thus

$$
\mathbb{P}(\mathcal{E}(\omega) \subset \mathcal{A}(\omega)) \geq \mathbb{P}\left(\mathcal{E}(\omega) \subset \Lambda_{K_{\varepsilon}}(\omega)\right)>1-\varepsilon
$$

from where we get that $\mathbb{P}(\mathcal{E}(\omega) \subset \mathcal{A}(\omega))=1$.

This means that the random attractor is the maximal negatively invariant compact random set, so that if two different random attractors exist, $\mathcal{A}_{1}(\omega), \mathcal{A}_{2}(\omega)$, we have that

$$
\mathbb{P}\left(\mathcal{A}_{1}(\omega) \subset \mathcal{A}_{2}(\omega)\right)=1=\mathbb{P}\left(\mathcal{A}_{2}(\omega) \subset \mathcal{A}_{1}(\omega)\right){ }^{1}
$$

On the other hand, the set $\mathcal{A}^{\prime}(\omega)=G\left(t, \theta_{-t} \omega\right) \mathcal{A}\left(\theta_{-t} \omega\right)$ is negatively invariant, since

$$
\begin{gathered}
\mathcal{A}^{\prime}\left(\theta_{t} \omega\right)=G(t, \omega) \mathcal{A}(\omega) \subset G(t, \omega) G\left(t, \theta_{-t} \omega\right) \mathcal{A}\left(\theta_{-t} \omega\right) \\
=G(t, \omega) \mathcal{A}^{\prime}(\omega) .
\end{gathered}
$$

Moreover, the set $\mathcal{A}^{\prime}(\omega)$ is $\mathbb{P}-$ a.s. compact. This follows from the fact that $\mathcal{A}\left(\theta_{-t} \omega\right)$ is $\mathbb{P}$ - a.s. compact, the map $x \mapsto G(t, \omega) x$ is upper semicontinuous and $G$ has compact values (see Aubin and Cellina, [3, p.42], Proposition 3). Then being $\mathcal{A}(\omega)$ the maximal negatively invariant compact set, $\mathcal{A}^{\prime}(\omega) \subset \mathcal{A}(\omega)$, so that $\mathcal{A}(\omega)$ is strictly invariant.

For the minimality, if $K(\omega)$ is another attracting random closed set, it is clear that $\Lambda_{D}(\omega) \subset K(\omega)$, for all bounded $D \subset X$. Indeed, if $y \in \Lambda_{D}(\omega)$ and $y \notin K(\omega)$, as this last set is closed, there exists $\delta>0$ such that

$$
\operatorname{dist}(y, K(\omega))>\delta
$$

On the other hand, there exists $t_{n} \nearrow+\infty, y_{n} \in G\left(t_{n}, \theta_{-t_{n}} \omega\right) D$ such that $\lim _{n \rightarrow \infty} y_{n}=$ $y$. But since $K(\omega)$ is attracting, there exists $n_{0}(\omega)$ such that for all $n \geq n_{0}$

$$
\operatorname{dist}\left(G\left(t_{n}, \theta_{-t_{n}} \omega\right) D, K(\omega)\right)<\frac{\delta}{2}
$$

so that

$$
\operatorname{dist}\left(y_{n}, K(\omega)\right)<\frac{\delta}{2}, \text { for all } n \geq n_{0},
$$

which contradicts that $y$ is the limit of $\left\{y_{n}\right\}$.

Thus, $\mathcal{A}(\omega) \subset K(\omega)$ and $\mathcal{A}(\omega)$ is minimal.

\footnotetext{
${ }^{1}$ In Flandoli and Schmalfuss [15] and Schenk-Hoppé [21] the attraction property is written with respect to random sets $\{D(\omega)\}$ fulfilling particular growth conditions. In our case, this would lead to a direct proof of uniqueness as it is shown in [14]. However, even with the weaker notion of attraction we use we also reach the uniqueness property.
} 
Finally, let us prove the measurability of the global attractor. First, we note that for any $x \in X$ and any nonrandom bounded $B \subset X$ the map

$$
(t, \omega) \mapsto \operatorname{dist}\left(x, G\left(t, \theta_{-t} \omega\right) B\right)
$$

is measurable. Indeed, since the map $(t, \omega) \mapsto \overline{G(t, \omega) B}$ is measurable, for any $a \in \mathbb{R}$ the set

$$
\mathcal{B}=\{(t, \xi): \operatorname{dist}(x, G(t, \xi) B)<a\}
$$

is measurable. Hence, being the map $v:(t, \omega) \mapsto\left(t, \theta_{t} \omega\right)$ measurable the set

$$
\mathcal{C}=v^{-1}(\mathcal{B})=\left\{(t, \omega): \xi=\theta_{t} \omega,(t, \xi) \in \mathcal{B}\right\}
$$

is measurable, so that the assertion follows.

Now, for each $\tau \geq 0$ we have

$$
\operatorname{dist}\left(x, \cup_{t \geq \tau} G\left(t, \theta_{-t} \omega\right) B\right)=\inf _{t \geq \tau} \operatorname{dist}\left(x, G\left(t, \theta_{-t} \omega\right) B\right) .
$$

Let $\pi_{\Omega}$ be the canonical projection of $\mathbb{R}_{+} \times \Omega$ onto $\Omega$. For an arbitrary $a \in \mathbb{R}$ we have

$$
\left\{\omega \in \Omega: \inf _{t \geq \tau} \operatorname{dist}\left(x, G\left(t, \theta_{-t} \omega\right) B\right)<a\right\}=\pi_{\Omega}\left\{(t, \omega): \operatorname{dist}\left(x, G\left(t, \theta_{-t} \omega\right) B\right)<a, t \geq \tau\right\} .
$$

Then, the measurability of $\omega \mapsto \operatorname{dist}\left(x, \overline{\cup_{t \geq \tau} G\left(t, \theta_{-t} \omega\right) B}\right)$ with respect to the $\mathbb{P}$-completion of $\mathcal{F}$ follows from the projection theorem (see Castaing and Valadier [10], Theorem III.23). Taking

$$
\mathcal{A}(\omega)=\overline{\cup_{m} \subset X} \cap_{\tau_{n} \geq 0} \overline{\cup_{t \geq \tau_{n}} G\left(t, \theta_{-t} \omega\right) B_{m}}
$$

over a countable number of $\tau_{n}$ and $B_{m}$ bounded we obtain that $\mathcal{A}$ is measurable with respect to the $\mathbb{P}$-completion of $\mathcal{F}$ (see Aubin and Frankowska [4], Theorem 8.2.4.).

Finally, thanks to Lemma 3.7 in Crauel [12] there exists a compact random set $\widetilde{\mathcal{A}}(\omega)$ measurable with respect to $\mathcal{F}$ such that $\widetilde{\mathcal{A}}(\omega)=\mathcal{A}(\omega)$ for $\mathbb{P}-$ a.a. $\omega \in \Omega$.

Remark 6 If instead of having compact values we assume that $x \mapsto G(t, \omega) x$ is lower semicontinuous, then the statement of the theorem remains true.

Indeed, the assumption of having compact values is only used to prove that $\mathcal{A}(\omega)$ is positively invariant (that is, $G(t, \omega) \mathcal{A}(\omega) \subset \mathcal{A}\left(\theta_{t} \omega\right)$ ). Take $y \in \mathcal{A}(\omega)$. There exists a sequence $D_{n} \subset X$ and $y_{n}$ such that $y_{n} \in \Lambda_{D_{n}}(\omega)$ and $y=\lim _{n \rightarrow+\infty} y_{n}$. By the lower semicontinuity of $G$, for any $z \in G(t, \omega) y$, there exist $z_{n}, z_{n} \in G(t, \omega) y_{n}$, with

$$
z=\lim _{n \rightarrow+\infty} z_{n}
$$

But note that, by the strict invariance of the limit sets (see Proposition 2), $z_{n} \in G(t, \omega) y_{n} \subset$ $\Lambda_{D_{n}}\left(\theta_{t} \omega\right)$, for all $n \in \mathbb{N}$, from which we get that $z \in \mathcal{A}\left(\theta_{t} \omega\right)$, and so $G(t, \omega) y \subset \mathcal{A}\left(\theta_{t} \omega\right)$. 
Remark 7 Notice that if $x \mapsto G(t, \omega) x$ is lower semicontinuous, then the map $(t, \omega) \mapsto$ $\overline{G(t, \omega) D}$ is measurable for all deterministic bounded sets $D \subset X, t \geq 0$ and $\mathbb{P}-$ a.a. $\omega \in \Omega$.

Indeed, if $x \mapsto G(t, \omega) x$ is lower semicontinuous, then

$$
(t, \omega) \mapsto \operatorname{dist}\left(x, G\left(t, \theta_{-t} \omega\right) D\right)=\inf _{y \in D} \operatorname{dist}\left(x, G\left(t, \theta_{-t} \omega\right) y\right),
$$

is measurable. Since the map $(t, \omega) \mapsto G(t, \omega) y$ is measurable, for any $a \in \mathbb{R}$ the set

$$
\mathcal{B}=\{(t, \xi): \operatorname{dist}(x, G(t, \xi) y)<a\}
$$

is measurable. Hence, being the map $v:(t, \omega) \mapsto\left(t, \theta_{t} \omega\right)$ measurable, the set

$$
\mathcal{C}=v^{-1}(\mathcal{B})=\left\{(t, \omega): \xi=\theta_{t} \omega,(t, \xi) \in \mathcal{B}\right\}
$$

is measurable. We have proved that the map $(t, \omega) \mapsto \operatorname{dist}\left(x, G\left(t, \theta_{-t} \omega\right) y\right)$ is measurable for any $y \in D$. Being $X$ separable, we can find a dense countable set $\left\{y_{n}\right\}, n \geq 1$, of the set $D$. In view of the lower semicontinuity of $G$, for any $z \in G\left(t, \theta_{-t} \omega\right) y, y \in D$, there exist sequences $y_{j}, z_{j} \in G\left(t, \theta_{-t} \omega\right) y_{j}$, such that $y_{j} \rightarrow y, z_{j} \rightarrow z$. Then

$$
\inf _{y \in D} \operatorname{dist}\left(x, G\left(t, \theta_{-t} \omega\right) y\right)=\inf _{n \geq 1} \operatorname{dist}\left(x, G\left(t, \theta_{-t} \omega\right) y_{n}\right)
$$

which proves the measurability of $(t, \omega) \mapsto \operatorname{dist}\left(x, G\left(t, \theta_{-t} \omega\right) D\right)$.

Remark 8 a) Clearly, if $X$ is a Banach space, we can also write $\mathcal{A}\left(\theta_{t} \omega\right)=\overline{\cup_{n \geq 1} \Lambda_{B_{n}}(\omega)}$, where $B_{n}$ are balls in $X$ centered at 0 with radius $n$.

b) The previous result is also true under weaker conditions, as we can suppose the existence of a compact attracting set $B(\omega)$ instead of a compact absorbing set. Some small changes would appear in the proof. But in the applications given in this paper we will find the conditions of the previous theorem.

\section{Attractors for stochastic differential inclusions}

\subsection{Setting of the problem}

Now, let $X$ be a real separable Hilbert space with the scalar product $\langle\cdot, \cdot\rangle$ and the norm $\|\cdot\|$. Consider the following stochastic differential inclusion

$$
\left\{\begin{array}{c}
\frac{d u}{d t} \in A u(t)+F(u(t))+\sum_{i=1}^{m} \phi_{i} \frac{d w_{i}(t)}{d t}, t \in(0, T), \\
u(0)=u_{0}
\end{array}\right.
$$


where $A: D(A) \rightarrow X$ is a linear operator, $\phi_{i} \in D(A)$ and $w_{i}(t)$ are independent twosided, i.e. $t \in \mathbb{R}$, real Wiener processes with $w_{i}(0)=0, i=1, \ldots, m$.

Let us introduce the next conditions:

(A) The operator $A$ is m-dissipative, i.e. $\forall y \in D(A)$

$$
<A y, y>\leq 0
$$

and $\operatorname{Im}(A-\lambda I)=X, \forall \lambda>0$.

(F1) $F: X \rightarrow C_{v}(X)$, where $C_{v}(X)$ is the set of all non-empty, bounded, closed, convex subsets of $X$.

(F2) The map $F$ is Lipschitz on $\overline{D(A)}$, i.e. $\exists C \geq 0$ such that $\forall y_{1}, y_{2} \in \overline{D(A)}$

$$
\operatorname{dist}_{H}\left(F\left(y_{1}\right), F\left(y_{2}\right)\right) \leq C\left\|y_{1}-y_{2}\right\|
$$

where $\operatorname{dist}_{H}(\cdot, \cdot)$ denotes the Hausdorff metric of bounded sets, i.e. $\operatorname{dist}_{H}(A, B)=$ $\max \{\operatorname{dist}(A, B), \operatorname{dist}(B, A)\}$.

Let $\zeta(t)=\sum_{i=1}^{m} \phi_{i} w_{i}(t)$. Let us consider the Wiener probability space $(\Omega, \mathcal{F}, \mathbb{P})$ defined by

$$
\Omega=\left\{\omega=\left(w_{1}(\cdot), \ldots, w_{m}(\cdot)\right) \in C\left(\mathbb{R}, \mathbb{R}^{m}\right) \mid \omega(0)=0\right\},
$$

equipped with the Borel $\sigma$-algebra $\mathcal{F}$, the Wiener measure $\mathbb{P}$, and the usual uniform convergence on bounded sets of $\mathbb{R}$. Each $\omega \in \Omega$ generates a map $\zeta(\cdot)=\sum_{i=1}^{m} \phi_{i} w_{i}(\cdot) \in$ $C(\mathbb{R}, X)$ such that $\zeta(0)=0$.

We make the change of variable $v(t)=u(t)-\zeta(t)$. Inclusion (3) turns into

$$
\left\{\begin{array}{c}
\frac{d v}{d t} \in A v(t)+F(v(t)+\zeta(t))+\sum_{i=1}^{m} A \phi_{i} w_{i}(t), \\
v(0)=v_{0}=u_{0} .
\end{array}\right.
$$

Remark 9 Note that (4) is a nonautonomous PDE parameterized by $\omega \in \Omega$. It would be possible to prove that this differential equation defines a nonautonomous multivalued dynamical system and then show the existence of a unique nonautonomous attractor for it, which would be equivalent to the existence of a unique random attractor for (3). However, to follow the general presentation in the previous section for general stochastic partial differential inclusions, we will follow the ideas and notation written there.

We shall define the multivalued map $\widetilde{F}:[0, T] \times \Omega \times X \rightarrow C_{v}(X)$,

$$
\widetilde{F}(t, \omega, x)=F(x+\zeta(t))+A \zeta(t) .
$$


It is easy to obtain from (F2) the existence of constants $D_{1}, D_{2}$ such that

$$
\|F(x)\|^{+} \leq D_{1}+D_{2}\|x\|
$$

where $\|F(x)\|^{+}=\sup _{y \in F(x)}\|y\|$. Hence,

$$
\|\widetilde{F}(t, \omega, x)\|^{+} \leq D_{1}+D_{2}\|x\|+D_{2}\|\zeta(t)\|+\|A \zeta(t)\|=n(t, \omega, x) .
$$

It follows that $\widetilde{F}$ satisfies the next property:

(F3) For any $x \in X$ there exists $n(\cdot) \in L_{1}(0, T)$ depending on $x$ and $\omega$ such that

$$
\|\widetilde{F}(t, \omega, x)\|^{+} \leq n(t) \text {, a.e. in }(0, T) .
$$

On the other hand, it is clear that $\widetilde{F}$ satisfies conditions $(F 1)-(F 2)$ for any fixed $t \in[0, T]$ and $\omega \in \Omega$, where the constant $C$ does not depend on $t$ or $\omega$.

Consider also the equation

$$
\left\{\begin{array}{c}
\frac{d v(t)}{d t}=A v(t)+f(t) \\
v(0)=v_{0}
\end{array}\right.
$$

where $f(\cdot) \in L_{1}([0, T], X)$.

Definition 5 The function $u:[0, T] \rightarrow X$ is called a strong solution of problem (5) if:

1. $u(\cdot)$ is continuous on $[0, T]$ and $u(0)=u_{0}$;

2. $u(\cdot)$ is absolutely continuous on any compact subset of $(0, T)$ and almost everywhere (a.e.) differentiable on $(0, T)$;

3. $u(\cdot)$ satisfies (5) a.e. on $(0, T)$.

Definition 6 The continuous function $v:[0, T] \rightarrow X$ is called an integral solution of problem (5) if:

1. $v(0)=v_{0}$;

2. $\forall \xi \in D(A)$,

$$
\|v(t)-\xi\|^{2} \leq\|v(s)-\xi\|^{2}+2 \int_{s}^{t}<f(\tau)+A \xi, v(\tau)-\xi>d \tau, t \geq s .
$$


Remark 10 We note that if $u(\cdot)$ is a strong solution of (5), then multiplying (5) by $v(t)-\xi$ and using (A), we obtain (6) after integration. This motivates the previous definition.

It is well known (see Barbu [7, p.124]) that any strong solution of problem (5) is an integral solution.

Definition 7 The process $v:[0, T] \times \Omega \rightarrow X$ is said to be an integral solution of problem (4) if for any $\omega \in \Omega$ :

1. $v(\cdot)=v(\cdot, \omega):[0, T] \rightarrow X$ is continuous.

2. $v(0)=v_{0}$;

3. For some selection $f \in L_{1}([0, T], X), f(t) \in \widetilde{F}(t, \omega, v(t))$ a.e. on $(0, T)$, the inequality (6) holds.

In what follows, we will omit $\omega$ if no confusion is possible.

If condition $(A)$ holds and $f \in L_{1}([0, T], X)$, then $\forall v_{0} \in \overline{D(A)}$ there exists a unique integral solution $v(\cdot)$ of (5) for each $T>0$ (see Barbu [7, p.124]). We shall denote this solution by $v(\cdot)=I\left(v_{0}\right) f(\cdot)$. Moreover, for any integral solutions $v_{i}(\cdot)=I\left(v_{i 0}\right) f_{i}(\cdot)$, $i=1,2$, the next inequality holds:

$$
\left\|v_{1}(t)-v_{2}(t)\right\| \leq\left\|v_{1}(s)-v_{2}(s)\right\|+\int_{s}^{t}\left\|f_{1}(\tau)-f_{2}(\tau)\right\| d \tau, t \geq s .
$$

If $(A),(F 1)-(F 3)$ hold, then $\forall v_{0} \in \overline{D(A)}$ there exists at least one integral solution of (4) for each $T>0$ (see Tolstonogov [24], Theorem 3.1). Moreover, for any $z(\cdot)=I\left(z_{0}\right) g(\cdot)$, $g(\cdot) \in L_{1}([0, T], X)$, and any $v_{0} \in \overline{D(A)}$ there exists an integral solution $v(\cdot)=I\left(v_{0}\right) f(\cdot)$ of (4) such that

$$
\begin{gathered}
\|v(t)-z(t)\| \leq \xi(t), \forall t \in[0, T], \\
\|f(t)-g(t)\| \leq \rho(t)+2 C \xi(t), \text { a.e. on }(0, T),
\end{gathered}
$$

where

$$
\begin{gathered}
\rho(t)=2 \operatorname{dist}(g(t), \widetilde{F}(t, \omega, z(t))) \\
\xi(t)=\left\|v_{0}-z_{0}\right\| \exp (2 C t)+\int_{0}^{t} \exp (2 C(t-s)) \rho(s) d s .
\end{gathered}
$$




\subsection{Construction of the MRDS}

Since $T>0$ is arbitrary, each solution can be extended on $[0, \infty)$. Let us denote by $\mathcal{D}\left(v_{0}, \omega\right)$ the set of all integral solutions of $(4)$ such that $v(0)=v_{0}$. We define the maps $G: \mathbb{R}^{+} \times \Omega \times \overline{D(A)} \rightarrow P(\overline{D(A)}), \theta_{s}: \Omega \rightarrow \Omega$ as follows

$$
\begin{gathered}
G(t, \omega) v_{0}=\left\{v(t)+\zeta(t) \mid v(\cdot) \in \mathcal{D}\left(v_{0}, \omega\right)\right\} \\
\theta_{s} \omega=\left(w_{1}(s+\cdot)-w_{1}(s), \ldots, w_{m}(s+\cdot)-w_{m}(s)\right) \in \Omega .
\end{gathered}
$$

Then the function $\widetilde{\zeta}$ corresponding to $\theta_{s} \omega$ is defined by $\widetilde{\zeta}(\tau)=\zeta(s+\tau)-\zeta(s)=$ $\sum_{i=1}^{m} \phi_{i}\left(w_{i}(s+\tau)-w_{i}(s)\right)$.

Proposition 4 Let $(A),(F 1),(F 2)$ hold. Then $G$ satisfies the cocycle property

$$
G\left(t_{1}+s, \omega\right) x=G\left(t_{1}, \theta_{s} \omega\right) G(s, \omega) x, \forall t_{1}, s \geq 0, x \in X, \omega \in \Omega
$$

Proof. First let $y \in G\left(t_{1}+s, \omega\right) x$. Then $y=y\left(t_{1}+s\right)=v\left(t_{1}+s\right)+\zeta\left(t_{1}+s\right)$, where $v(\cdot) \in \mathcal{D}(x, \omega)$. It is clear that $y(s) \in G(s, \omega) x$. We have to prove that $y \in$ $G\left(t_{1}, \theta_{s} \omega\right) y(s)$. Define $z(t)=y(t+s)-\zeta(t+s)+\zeta(s), \forall t \geq 0, g(t)=f(t+s)-A \zeta(s)$, a.e. $t>0$, where $y(t)-\zeta(t)=v(t)=I(x) f(t)$. For any $r \leq t, \xi \in D(A)$ we obtain

$$
\begin{gathered}
\|z(t)-\xi\|^{2}=\|v(t+s)+\zeta(s)-\xi\|^{2} \leq\|v(r+s)+\zeta(s)-\xi\|^{2} \\
+2 \int_{r}^{t}\langle f(\tau+s)+A \xi-A \zeta(s), v(\tau+s)+\zeta(s)-\xi\rangle d \tau \\
=\|z(r)-\xi\|^{2}+2 \int_{r}^{t}\langle g(\tau)+A \xi, z(\tau)-\xi\rangle d \tau .
\end{gathered}
$$

On the other hand,

$$
\begin{gathered}
g(t) \in F(v(t+s)+\zeta(t+s))+A \zeta(t+s)-A \zeta(s) \\
=F(z(t)+\zeta(t+s)-\zeta(s))+A(\zeta(t+s)-\zeta(s))=\widetilde{F}\left(t, \theta_{s} \omega, z(t)\right) .
\end{gathered}
$$

Therefore, since $z(0)=y(s)$, it follows that $z(\cdot) \in \mathcal{D}\left(y(s), \theta_{s} \omega\right)$. Being $y=y\left(t_{1}+s\right)=$ $z\left(t_{1}\right)+\zeta\left(t_{1}+s\right)-\zeta(s)$, we get $y \in G\left(t_{1}, \theta_{s} \omega\right) y(s)$. Hence,

$$
G\left(t_{1}+s, \omega\right) x \subset G\left(t_{1}, \theta_{s} \omega\right) G(s, \omega) x .
$$

Conversely, let $y \in G\left(t_{1}, \theta_{s} \omega\right) G(s, \omega) x$. Then there exist $v_{1}(\cdot) \in \mathcal{D}(x, \omega)$ and $v_{2}(\cdot) \in$ $\mathcal{D}\left(y_{1}(s), \theta_{s} \omega\right), y_{1}(s)=v_{1}(s)+\zeta(s)$, such that $y=v_{2}\left(t_{1}\right)+\zeta\left(t_{1}+s\right)-\zeta(s)$. Let

$$
z(t)=\left\{\begin{array}{c}
v_{1}(t), \text { if } 0 \leq t \leq s \\
v_{2}(t-s)-\zeta(s), \text { if } s \leq t
\end{array}\right.
$$




$$
f(t)=\left\{\begin{array}{c}
f_{1}(t), \text { if } 0 \leq t \leq s \\
f_{2}(t-s)+A \zeta(s), \text { if } s \leq t
\end{array}\right.
$$

where $v_{1}(\cdot)=I(x) f_{1}(\cdot), v_{2}(\cdot)=I\left(y_{1}(s)\right) f_{2}(\cdot)$. We have to check that for a.a. $t \in(0, T)$, $f(t) \in F(z(t)+\zeta(t))+A \zeta(t)$. If $t \leq s$ it is obvious that $f(t) \in F\left(v_{1}(t)+\zeta(t)\right)+$ $A \zeta(t)=F(z(t)+\zeta(t))+A \zeta(t)$. If $t \geq s$ we have

$$
\begin{gathered}
f(t)=f_{2}(t-s)+A \zeta(s) \in F\left(v_{2}(t-s)+\zeta(t)-\zeta(s)\right)+A \zeta(t) \\
=F(z(t)+\zeta(t))+A \zeta(t)=\widetilde{F}(t, \omega, z(t)) .
\end{gathered}
$$

It remains to prove that $z(\cdot)$ satisfies (6) for any $r \leq t$. If $t \leq s$ the inequality is evident. If $r<s<t$ we get

$$
\begin{gathered}
\|z(t)-\xi\|^{2}=\left\|v_{2}(t-s)-\zeta(s)-\xi\right\|^{2} \leq\left\|v_{2}(0)-\zeta(s)-\xi\right\|^{2} \\
+2 \int_{s}^{t}\left\langle f_{2}(\tau-s)+A \zeta(s)+A \xi, v_{2}(\tau-s)-\zeta(s)-\xi\right\rangle d \tau \\
\quad \leq\left\|v_{1}(r)-\xi\right\|^{2}+2 \int_{r}^{s}\left\langle f_{1}(\tau)+A \xi, v_{1}(\tau)-\xi\right\rangle d \tau \\
+2 \int_{s}^{t}\left\langle f_{2}(\tau-s)+A \zeta(s)+A \xi, v_{2}(\tau-s)-\zeta(s)-\xi\right\rangle d \tau \\
=\|z(r)-\xi\|^{2}+2 \int_{r}^{t}\langle f(\tau)+A \xi, z(\tau)-\xi\rangle d \tau .
\end{gathered}
$$

Finally, the case $s \leq r$ is rather similar. Therefore, $z(\cdot) \in \mathcal{D}(x, \omega)$ and

$$
y=v_{2}\left(t_{1}\right)+\zeta\left(t_{1}+s\right)-\zeta(s)=z\left(t_{1}+s\right)+\zeta\left(t_{1}+s\right) \in G\left(t_{1}+s, \omega\right) x .
$$

Hence,

$$
G\left(t_{1}, \theta_{s} \omega\right) G(s, \omega) x \subset G\left(t_{1}+s, \omega\right) x .
$$

Lemma 5 Let $(A),(F 1),(F 2)$ hold. Then, for any fixed $t \geq 0$ and $\omega \in \Omega$,

$$
\operatorname{dist}_{H}(G(t, \omega) x, G(t, \omega) y) \leq \exp (2 C t)\|x-y\|, \forall x, y \in \overline{D(A)} .
$$

Proof. Let $\mu \in G(t, \omega) y, t \geq 0$, be arbitrary. Then there exists $v(\cdot)=I(y) f(\cdot)$, an integral solution of (4), such that $\mu=v(t)+\zeta(t)$. Since $f(\tau) \in \widetilde{F}(\tau, \omega, v(\tau))$, a.e. on $(0, t)$, it follows that $\operatorname{dist}(f(\tau), \widetilde{F}(\tau, \omega, v(\tau)))=0$, a.e. on $(0, t)$. Then in view of $(8)$ there exists an integral solution $u(\cdot)=I(x) g(\cdot)$ of $(4)$ such that

$$
\|u(\tau)-v(\tau)\| \leq \exp (2 C \tau)\|x-y\| \text {, on }[0, t]
$$

It is clear that $\widetilde{\mu}=u(t)+\zeta(t) \in G(t, \omega) x$ and

$$
\|\mu-\widetilde{\mu}\| \leq \exp (2 C t)\|x-y\|
$$


Since $\mu \in G(t, \omega) y$ is arbitrary, we get

$$
\operatorname{dist}(G(t, \omega) y, G(t, \omega) x) \leq \exp (2 C t)\|x-y\| \text {. }
$$

In the same way we can prove the converse $\operatorname{dist}(G(t, \omega) x, G(t, \omega) y) \leq \exp (2 C t)\|x-y\|$. Hence, the inequality (10) holds.

Lemma 6 Let $(A),(F 1),(F 2)$ hold. Then for any fixed $t \geq 0, x \in \overline{D(A)}$,

$$
\operatorname{dist}_{H}\left(G\left(t, \omega_{1}\right) x, G\left(t, \omega_{2}\right) x\right) \leq k(t)\left\|\omega_{1}-\omega_{2}\right\|_{C\left([0, t], \mathbb{R}^{m}\right)}, \text { for } \omega_{1}, \omega_{2} \in \Omega,
$$

where $k(t)$ is increasing and continuous.

Proof. Let $\zeta^{1}, \zeta^{2} \in C(\mathbb{R}, X)$ be the maps corresponding to $\omega_{1}$ and $\omega_{2}$, respectively. Let $y \in G\left(t, \omega_{1}\right) x$ be arbitrary. Then $y=y(t)+\zeta^{1}(t), y(\cdot)=I(x) f_{0}(\cdot)$ and

$$
f_{0}(\tau) \in F\left(y(\tau)+\zeta^{1}(\tau)\right)+A \zeta^{1}(\tau)=\widetilde{F}\left(\tau, \omega_{1}, y(\tau)\right) \text {, a.e. on }(0, t) \text {. }
$$

Now by $(F 2)$,

$$
\begin{gathered}
\rho(\tau)=2 \operatorname{dist}\left(f_{0}(\tau), \widetilde{F}\left(\tau, \omega_{2}, y(\tau)\right)\right) \\
\leq 2\left(\left\|A \zeta^{1}(\tau)-A \zeta^{2}(\tau)\right\|+\operatorname{dist}\left(F\left(y(\tau)+\zeta^{1}(\tau)\right), F\left(y(\tau)+\zeta^{2}(\tau)\right)\right)\right) \\
\leq 2\left(\left\|A \zeta^{1}(\tau)-A \zeta^{2}(\tau)\right\|+C\left\|\zeta^{1}(\tau)-\zeta^{2}(\tau)\right\|\right) \\
\leq 2 K\left(\sum_{i=1}^{m}\left(\left\|A \phi_{i}\right\|+\left\|\phi_{i}\right\|\right)\right)\left\|\omega_{1}-\omega_{2}\right\|_{C\left([0, t], \mathbb{R}^{m}\right)} .
\end{gathered}
$$

In view of inequalities (8)-(9) there exists an integral solution of $(4), v(\cdot)=I(x) f(\cdot)$, $f(\tau) \in F\left(v(\tau)+\zeta^{2}(\tau)\right)+A \zeta^{2}(\tau)=\widetilde{F}\left(\tau, \omega_{2}, v(\tau)\right)$, a.e. on $(0, t)$, such that

$$
\begin{gathered}
\|v(t)-y(t)\| \leq \int_{0}^{t} \exp (2 C(t-\tau)) \rho(\tau) d \tau \\
\leq 2 K \exp (2 C t) t\left(\sum_{i=1}^{m}\left(\left\|A \phi_{i}\right\|+\left\|\phi_{i}\right\|\right)\right)\left\|\omega_{1}-\omega_{2}\right\|_{C\left([0, t], \mathbb{R}^{m}\right)} .
\end{gathered}
$$

Let $v=v(t)+\zeta^{2}(t) \in G\left(t, \omega_{2}\right) x$. Then

$$
\begin{gathered}
\|y-v\| \leq\|v(t)-y(t)\|+\left\|\zeta^{1}(t)-\zeta^{2}(t)\right\| \\
\leq \widetilde{K}(\exp (2 C t) t+1)\left(\sum_{i=1}^{m}\left(\left\|A \phi_{i}\right\|+\left\|\phi_{i}\right\|\right)\right)\left\|\omega_{1}-\omega_{2}\right\|_{C\left([0, t], \mathbb{R}^{m}\right)} .
\end{gathered}
$$

Repeating the same argument for any $y \in G\left(t, \omega_{2}\right) x$ we finally have the existence of a continuous increasing function $k(t)$ such that

$$
\operatorname{dist}_{H}\left(G\left(t, \omega_{1}\right) x, G\left(t, \omega_{2}\right) x\right) \leq k(t)\left\|\omega_{1}-\omega_{2}\right\|_{C\left([0, t], \mathbb{R}^{m}\right)} .
$$


Let $C([0, T], X)$ (respectively $C([0,+\infty), X))$ be the Banach space of continuous functions from $[0, T]$ (respectively $[0,+\infty))$ into $X$. By $\pi_{t}: C([0, \infty), X) \rightarrow C([0, T], X)$ we denote the projection operator

$$
\pi_{T}(y(\cdot))=\{\widetilde{y}(\cdot) \in C([0, T], X) \mid \widetilde{y}(s)=y(s), \forall t \in[0, T]\} .
$$

Lemma 7 Let $(A),(F 1),(F 2)$ hold and the semigroup $S(t, \cdot)$ generated by the operator $A$ be compact. Then, the semiflow $G$ has compact values.

Proof. From Tolstonogov [24, Theorem 3.4], it follows that for each $v_{0} \in \overline{D(A)}, T>0$ the set $\pi_{T} \mathcal{D}\left(v_{0}, \omega\right)$ is compact in $C([0, T], X)$. Hence, the set $G(T, \omega) v_{0}$ is compact in $X$.

Theorem 8 Let $(A),(F 1),(F 2)$ hold and the semigroup $S(t, \cdot)$ generated by the operator $A$ be compact. Then, $G$ generates a MRDS.

Proof. It is evident from the definition of the map $G$ that $t \mapsto G(t, \omega) x$ is lower semicontinuous. Let us prove that the map $(t, \omega, x) \mapsto G(t, \omega) x$ is lower semicontinuous. Let $t_{n} \rightarrow t, \omega_{n} \rightarrow \omega, x_{n} \rightarrow x$. We take an arbitrary $y \in G(t, \omega) x$. For any $\varepsilon>0$ there exists $\delta_{1}>0$ such that if $\left|t_{n}-t\right|<\delta_{1}$ then for some $y_{n} \in G\left(t_{n}, \omega\right) x,\left\|y_{n}-y\right\|<\varepsilon$. On the other hand, it follows from Lemmas 5-6 that the map $(\omega, x) \longmapsto G(t, \omega) x$ is lower semicontinuous uniformly for $t$ in bounded sets. Hence there exists $\delta_{2}>0$ such that if $\left\|x_{n}-x\right\|<\delta_{2},\left\|\omega_{n}-\omega\right\|_{C\left(\mathbb{R}, \mathbb{R}^{m}\right)}<\delta_{2}$ then for any $t_{n}$ there is $v_{n} \in G\left(t_{n}, \omega_{n}\right) x_{n}$ for which $\left\|y_{n}-v_{n}\right\|<\varepsilon$. Therefore,

$$
\left\|y-v_{n}\right\| \leq\left\|y-y_{n}\right\|+\left\|y_{n}-v_{n}\right\| \leq 2 \varepsilon .
$$

Now we have to prove that the multivalued map $G: \mathbb{R}^{+} \times \Omega \times \overline{D(A)} \rightarrow C(\overline{D(A)})$ is measurable with respect to the $\sigma$-algebra $\mathcal{B}\left(\mathbb{R}^{+}\right) \otimes \mathcal{F} \otimes \mathcal{B}(\overline{D(A)})$. Since this $\sigma$-algebra contains all open sets of the complete separable metric space $\mathbb{R}^{+} \times \Omega \times \overline{D(A)}$ and, in view of the previous lemma, $G$ has closed values (in fact, compact values), the lower semicontinuity of $G$ implies the measurability of the semiflow. Indeed, the map $G$ is measurable if and only if the inverse image of any open set $\mathcal{O} \subset \overline{D(A)}$

$$
G^{-1}(\mathcal{O})=\left\{(t, \omega, x) \in \mathbb{R}_{+} \times \Omega \times \overline{D(A)}: G(t, \omega) x \cap \mathcal{O} \neq \emptyset\right\}
$$

is measurable (see Aubin and Frankowska, [4], Theorem 8.3.1.). Being the map $G$ lower semicontinuous, the inverse image of any open set is open and then measurable (see Aubin and Frankowska, [4, p.40]).

Finally, Proposition 4 implies that $G$ generates a MRDS.

Lemma 9 Let $(A),(F 1),(F 2)$ hold. Then for any $v_{0} \in \overline{D(A)}$ the set $\pi_{T}\left(\mathcal{D}\left(v_{0}, \omega\right)\right)$ is bounded in $C([0, T], X)$. For each $t \geq 0, v_{0} \in \overline{D(A)}$ the set $G(t, \omega) v_{0}$ is bounded. 
Proof. It follows from $(F 2)$ that there exist constants $D_{1}, D_{2} \geq 0$ such that $\forall x \in \overline{D(A)}$, $\forall y \in F(x)$,

$$
\|y\| \leq D_{1}+D_{2}\|x\| \text {. }
$$

We take a fixed $T>0$. We shall denote by $z(\cdot) \in C([0, T], X)$ the unique integral solution of the equation

$$
\left\{\begin{array}{c}
\frac{d z}{d t}=A(z(t)), 0 \leq t \leq T \\
z(0)=v_{0} .
\end{array}\right.
$$

Let $r_{0}=\max \{\|z(t)\|, 0 \leq t \leq T\}$ and let $r(\cdot) \in C([0, T])$ be the solution to the equation

$$
\left\{\begin{array}{c}
r^{\prime}(t)=D_{1}+D_{2} r(t), 0 \leq t \leq T \\
r(0)=r_{0} .
\end{array}\right.
$$

Consider an arbitrary solution $v(\cdot) \in \mathcal{D}\left(v_{0}, \omega\right), v(\cdot)=I\left(v_{0}\right) f(\cdot)$. Then it follows from $(7)$ and (12) that

$$
\begin{gathered}
\|v(t)\| \leq\|z(t)\|+\int_{0}^{t}\|f(\tau)\| d \tau \\
\leq r_{0}+\int_{0}^{t}\left(D_{1}+D_{2}(\|v(\tau)\|+\|\zeta(\tau)\|)+\|A \zeta(\tau)\|\right) d \tau, \forall t \leq T .
\end{gathered}
$$

Let $K(\omega)$ be such that

$$
\sup _{\tau \in[0, T]}\left\{D_{2}\|\zeta(\tau)\|+\|A \zeta(\tau)\|\right\} \leq K(\omega)
$$

Then

$$
\|v(t)\| \leq r_{0}+\left(D_{1}+K(\omega)\right) t+D_{2} \int_{0}^{t}\|v(\tau)\| d \tau .
$$

Using the Gronwall Lemma we have that for any $t \in[0, T]$ the next inequalities are satisfied:

$$
\left\{\begin{array}{c}
\|v(t)\| \leq\left(r_{0}+\frac{\widetilde{D}_{1}}{D_{2}}\right) \exp \left(D_{2} t\right)-\frac{\widetilde{D}_{1}}{D_{2}}=r(t, \omega), \text { if } D_{2} \neq 0 \\
\|v(t)\| \leq r_{0}+\widetilde{D}_{1} t, \text { if } D_{2}=0
\end{array}\right.
$$

where $\widetilde{D}_{1}=D_{1}+K(\omega)$. Hence, $\pi_{T} \mathcal{D}\left(v_{0}, \omega\right)$ is bounded in $C([0, T], X), \forall T \geq 0, \forall v_{0} \in$ $\overline{D(A)}$. It is obvious from the definition of $G$ that the set $G(t, \omega) v_{0}$ is bounded for each $t \geq 0, v_{0} \in \overline{D(A)}$.

For $T>0$ and bounded $B \subset \overline{D(A)}$, let us denote $\mathcal{D}(B, \omega)=\cup_{x \in B} \mathcal{D}(x, \omega)$ and

$$
M(B, \omega, T)=\left\{f(\cdot) \in L_{1}([0, T], X) \mid v(\cdot)=I(x) f(\cdot), v(\cdot) \in \pi_{T} \mathcal{D}(B, \omega)\right\}
$$

Lemma 10 Let $(A),(F 1),(F 2)$ hold. Then for any $T>0$ and any bounded set $B \subset$ $\overline{D(A)}$ the sets $M(B, \omega, T)$ and $\pi_{T} \mathcal{D}(B, \omega)$ are bounded in $L_{\infty}([0, T], X)$ and $C([0, T], X)$, respectively. 
Proof. Let $x \in B, T>0$, be arbitrary. In view of Lemma 9, there exists $K_{1}(\omega)>0$ such that for any $v(\cdot) \in \mathcal{D}(x, \omega)$,

$$
\|v(t)\| \leq K_{1}(\omega), \forall t \in[0, T] .
$$

We take an arbitrary $u(\cdot) \in \mathcal{D}(B, \omega), u(0)=y \in B$. Then, in the same way as in Lemma 5 , we obtain the existence of $v(\cdot) \in \mathcal{D}(x, \omega)$ such that

$$
\|v(t)-u(t)\| \leq \exp (2 C T)\|x-y\|, \text { on }[0, T] \text {. }
$$

Hence

$$
\|u(t)\| \leq\|v(t)\|+\exp (2 C T)\|x-y\| \leq K_{1}(\omega)+\exp (2 C T) K_{2} \text {, on }[0, T],
$$

where $K_{1}(\omega), K_{2}$ depend on $B$. We have proved that $\pi_{T} \mathcal{D}(B, \omega)$ is bounded in $C([0, T], X)$.

Further, we must prove that $M(B, \omega, T)$ is bounded in $L_{\infty}([0, T], X)$. Let $f(\cdot) \in$ $M(B, \omega, T)$ be arbitrary. Then, there exist $x \in B, x(\cdot) \in \mathcal{D}(x, \omega)$, such that $x(\cdot)=$ $I(x) f(\cdot), f(t) \in F(x(t)+\zeta(t))+A \zeta(t)$, a.e. on $(0, T)$. Let $\bar{x}$ be fixed. Since $F$ has bounded values, $\|\bar{y}\| \leq D<\infty, \forall \bar{y} \in F(\bar{x})$. For almost all $t \in(0, T)$ and any $\epsilon>0$, there exists $y_{\epsilon}(t) \in F(\bar{x})$ such that

$$
\operatorname{dist}(f(t)-A \zeta(t), F(\bar{x}))=\left\|f(t)-A \zeta(t)-y_{\epsilon}(t)\right\|-\epsilon .
$$

Then, it follows from $(F 2)$ that

$$
\begin{gathered}
\|f(t)\| \leq\left\|y_{\epsilon}(t)\right\|+\|A \zeta(t)\|+\epsilon+C\|x(t)+\zeta(t)-\bar{x}\|, \\
\leq D+\|A \zeta(t)\|+\epsilon+C\|x(t)+\zeta(t)-\bar{x}\|, \text { a.e. on }(0, T) .
\end{gathered}
$$

Since $\pi_{T} \mathcal{D}(B, \omega)$ is bounded in $C([0, T], X)$, we obtain the required result.

\subsection{Existence of the global random attractor}

In order to obtain the existence of a compact absorbing set we need more regularity of the integral solutions. Namely, we shall suppose that each integral solution of (4) is, in fact, a strong solution of (5).

Proposition 11 Let $(A),(F 1),(F 2)$ hold. Suppose that each integral solution of (4), $v(\cdot)=I\left(u_{0}\right) f(\cdot)$ is a strong solution of (5). Let there exist constants $\delta>0, M \geq 0$ such that $\forall u \in D(A), y \in F(u)$,

$$
\langle y, u\rangle \leq(-\delta+\varepsilon)\|u\|^{2}+M
$$

where $\varepsilon \geq 0$ is the biggest constant such that

$$
\langle A u, u\rangle \leq-\varepsilon\|u\|^{2}, \forall u \in D(A) .
$$


Then there exists a random radius $r(\omega)>0$ such that for $\mathbb{P}-$ almost all $\omega \in \Omega$ and any bounded set $B \subset \overline{D(A)}$ we can find $T(B)=T(B, \omega) \geq 1$ for which

$$
\left\|G\left(-1+t_{0}, \theta_{-t_{0}} \omega\right) u_{0}\right\|^{+} \leq r\left(\theta_{-1} \omega\right), \forall t_{0} \geq T(B), \forall u_{0} \in B .
$$

Remark 11 We observe that, since $A$ is m-dissipative, $\langle A u, u\rangle \leq 0, \forall u \in D(A)$.

Proof. We note that for any $y \in G\left(t, \theta_{s} \omega\right) u_{0}, y=v(t)+\zeta(t+s)-\zeta(s)$, being $v(\cdot)=I\left(u_{0}\right) f(\cdot)$ an integral solution of

$$
\left\{\begin{array}{c}
\frac{d v}{d t} \in A v(t)+F(v(t)+\zeta(t+s)-\zeta(s))+A \zeta(t+s)-A \zeta(s), \\
v(0)=u_{0},
\end{array}\right.
$$

which is a strong solution of (5) with

$$
f(t) \in F(v(t)+\zeta(t+s)-\zeta(s))+A \zeta(t+s)-A \zeta(s) \text {, a.e. in }(0, T) .
$$

After the change of variable $z(t)=v(t)-\zeta(s)$, we obtain that $y=z(t)+\zeta(t+s)$, being $z(\cdot)$ the integral solution (in fact, a strong one) of the problem

$$
\left\{\begin{array}{c}
\frac{d z}{d t}=A z(t)+g(t) \\
z(0)=u_{0}-\zeta(s)
\end{array}\right.
$$

where $g(t)=f(t)+A \zeta(s)=h(t)+A \zeta(t+s) \in F(z(t)+\zeta(t+s))+A \zeta(t+s)$, a.e. in $(0, T)$, and $h(t)=f(t)-A \zeta(t+s)+A \zeta(s)$.

In our case $s=-t_{0}$. Multiplying (16) by $z(t)$ we have

$$
\begin{gathered}
\frac{1}{2} \frac{d}{d t}\|z(t)\|^{2}=\langle A z(t), z(t)\rangle+\langle g(t), z(t)\rangle \\
=\langle A z(t), z(t)\rangle+\langle h(t), z(t)+\zeta(t+s)\rangle-\langle h(t), \zeta(t+s)\rangle+\langle A \zeta(t+s), z(t)\rangle .
\end{gathered}
$$

Now by (14), (15) we get

$$
\begin{gathered}
\frac{1}{2} \frac{d}{d t}\|z(t)\|^{2} \leq-\varepsilon\|z(t)\|^{2}+(\varepsilon-\delta)\left\|z(t)+\zeta\left(t-t_{0}\right)\right\|^{2} \\
+\left\|F\left(z(t)+\zeta\left(t-t_{0}\right)\right)\right\|^{+}\left\|\zeta\left(t-t_{0}\right)\right\|+\left\|A \zeta\left(t-t_{0}\right)\right\|\|z(t)\|+M .
\end{gathered}
$$

Inequality (12) and Young's inequality then imply

$$
\begin{gathered}
\frac{1}{2} \frac{d}{d t}\|z(t)\|^{2} \leq-\delta\|z(t)\|^{2}+(\varepsilon-\delta)\left\|\zeta\left(t-t_{0}\right)\right\|^{2} \\
+\left(D_{1}+D_{2}\|z(t)\|+D_{2}\left\|\zeta\left(t-t_{0}\right)\right\|\right)\left\|\zeta\left(t-t_{0}\right)\right\|+\frac{1}{\delta}\left\|A \zeta\left(t-t_{0}\right)\right\|^{2}+\frac{\delta}{4}\|z(t)\|^{2}+M
\end{gathered}
$$


$\leq-\frac{\delta}{2}\|z(t)\|^{2}+\left(\varepsilon-\delta+D_{2}+\frac{D_{2}^{2}}{\delta}\right)\left\|\zeta\left(t-t_{0}\right)\right\|^{2}+D_{1}\left\|\zeta\left(t-t_{0}\right)\right\|+\frac{1}{\delta}\left\|A \zeta\left(t-t_{0}\right)\right\|^{2}+M$.

Hence,

$$
\frac{d}{d t}\|z(t)\|^{2} \leq-\delta\|z(t)\|^{2}+p\left(t-t_{0}, \omega\right)
$$

where

$p\left(t-t_{0}, \omega\right)=2\left(\varepsilon-\delta+D_{2}+\frac{D_{2}^{2}}{\delta}\right)\left\|\zeta\left(t-t_{0}\right)\right\|^{2}+2 D_{1}\left\|\zeta\left(t-t_{0}\right)\right\|+\frac{2}{\delta}\left\|A \zeta\left(t-t_{0}\right)\right\|^{2}+2 M$.

Multiplying (17) by $\exp (\delta t)$ and integrating over $\left(0,-1+t_{0}\right)$ we obtain

$$
\begin{gathered}
\left\|z\left(-1+t_{0}\right)\right\|^{2} \leq \exp \left(-\delta\left(-1+t_{0}\right)\right)\|z(0)\|^{2} \\
+\exp \left(-\delta\left(-1+t_{0}\right)\right) \int_{0}^{-1+t_{0}} \exp (\delta s) p\left(s-t_{0}, \omega\right) d s
\end{gathered}
$$

and then, by the change $s-t_{0}=\tau$,

$$
\begin{aligned}
\left\|z\left(-1+t_{0}\right)\right\|^{2} \leq & \exp \left(-\delta\left(-1+t_{0}\right)\right)\left\|u_{0}\right\|^{2}+\exp \left(-\delta\left(-1+t_{0}\right)\right)\left\|\zeta\left(t_{0}\right)\right\|^{2} \\
& +\int_{-\infty}^{-1} \exp (-\delta(-1-\tau)) p(\tau, \omega) d \tau .
\end{aligned}
$$

We take

$$
\begin{gathered}
r_{1}^{2}\left(\theta_{-1} \omega\right)=1+\sup _{t_{0} \leq-1} \exp \left(-\delta\left(-1+t_{0}\right)\right)\left\|\zeta\left(t_{0}\right)\right\|^{2}+\int_{-\infty}^{-1} \exp (-\delta(-1-\tau)) p(\tau, \omega) d \tau, \\
r\left(\theta_{-1} \omega\right)=r_{1}\left(\theta_{-1} \omega\right)+\|\zeta(-1)\| .
\end{gathered}
$$

The radius $r\left(\theta_{-1} \omega\right)$ is $\mathbb{P}$-a.s. finite, because $\left\|\zeta\left(-t_{0}\right)\right\|^{2}$ and $p(\tau, \omega)$ have at most polynomial growth for $\mathbb{P}$-almost all $\omega \in \Omega$. For a bounded set $B$ we choose $T(B) \geq 1$ such that

$$
\exp \left(-\delta\left(-1+t_{0}\right)\right)\left\|u_{0}\right\|^{2} \leq 1, \forall t_{0} \geq T(B), \forall u_{0} \in B
$$

Since $y=z\left(-1+t_{0}\right)+\zeta(-1)$ we have

$$
\|y\| \leq\left\|z\left(-1+t_{0}\right)\right\|+\|\zeta(-1)\| \leq r\left(\theta_{-1} \omega\right),
$$

for $\mathbb{P}$-a.a. $\omega \in \Omega$ and any $y \in G\left(-1+t_{0}, \theta_{-t_{0}} \omega\right) u_{0}, u_{0} \in B$.

Theorem 12 Let the conditions of Proposition 11 hold, the semigroup $S(t, \cdot)$ generated by the operator $A$ be compact and the multivalued map $G(1, \omega)$ be compact (that is, it maps bounded sets into precompact ones). Then, $G$ has the minimal global random attractor $\mathcal{A}(\omega)$. Moreover, it is measurable with respect to $\mathcal{F}$. 
Proof. First, due to Lemma $5, G(t, \omega)$ is a continuous multivalued map in the sense of Hausdorff's metric. On the other hand, it follows from Lemma 7 that $G(t, \omega)$ has compact values. Hence, the map $G(t, \omega)$ is continuous (see Aubin and Cellina [3, p.67]) and, consequently, the multivalued semiflow $G(t, \omega)$ is upper and lower semicontinuous for any $t \geq 0$ and $\omega \in \Omega$.

Let us define the random ball

$$
B\left(r\left(\theta_{-1} \omega\right)\right)=\left\{u \in \overline{D(A)} \mid\|u\| \leq r\left(\theta_{-1} \omega\right)\right\}
$$

where $r\left(\theta_{-1} \omega\right)$ is taken from Proposition 11. Let

$$
K(\omega)=\overline{G\left(1, \theta_{-1} \omega\right) B\left(r\left(\theta_{-1} \omega\right)\right)} .
$$

The set $K(\omega)$ is $\mathbb{P}$-a.s. compact, since the operator $G\left(1, \theta_{-1} \omega\right)$ is compact. It follows from Proposition 11 that for any bounded nonrandom set $B$ and $\mathbb{P}$-a.a $\omega \in \Omega$ there exists such that $T(B)=T(B, \omega) \geq 1$ such that $\forall t_{0} \geq T(B)$,

$$
G\left(t_{0}, \theta_{-t_{0}} \omega\right) B \subset G\left(1, \theta_{-1} \omega\right) G\left(-1+t_{0}, \theta_{-t_{0}} \omega\right) B \subset K(\omega) .
$$

Therefore, $G$ has the compact random absorbing set $K(\omega)$. We note that being $(\omega, x) \mapsto$ $G\left(1, \theta_{-1} \omega\right) x$ a Carathéodory map, $K(\omega)$ is measurable with respect to the completion of $\mathcal{F}$ (see Aubin and Frankowska [4], Corollary 8.2.13. and Theorem 8.2.8.), and so with respect to $\mathcal{F}$ (Crauel [12], Lemma 3.7), although this fact is not necessary.

The statement of the theorem follows then from Theorem 3.

\section{Applications}

\subsection{The case of a subdifferential map}

In order to check when the operator $G(1, \omega)$ is compact we shall consider the case where the operator $-A$ is the subdifferential of a proper lower semicontinuous function $\varphi: X \rightarrow$ $(-\infty,+\infty]$. Inclusion (3) turns into

$$
\left\{\begin{array}{c}
\frac{d u}{d t} \in-\partial \varphi(u)+F(u)+\sum_{i=1}^{m} \phi_{i} \frac{d w_{i}(t)}{d t}, t \in[0, T] \\
u(0)=u_{0}
\end{array}\right.
$$

where $F: X \rightarrow 2^{X}$ is a multivalued map, satisfying $(F 1)-(F 2), \overline{D(A)}=\overline{D(\partial \varphi)}$. It is well known (see Barbu [7, p.54 and 71]) that $-\partial \varphi$ is an m-dissipative operator. Moreover, $\overline{D(\varphi)}=\overline{D(\partial \varphi)}$. Then, $\partial \varphi$ generates a nonlinear semigroup of operators $S(t, \cdot): \overline{D(\varphi)} \rightarrow$ $\overline{D(\varphi)}$ and the differential inclusion (18) gives the multivalued map $G(t, \omega): \overline{D(\varphi)} \rightarrow 2^{\overline{D(\varphi)}}$. It is known (see Haraux [16, p.1398]) that the semigroup $S$ is compact if the following condition is satisfied: 
(H) The level sets

$$
M_{R}=\{u \in D(\varphi) \mid\|u\| \leq R, \varphi(u) \leq R\}
$$

are compact in $X$ for any $R>0$.

If $(H)$ holds, it follows from Lemma 7 that $G$ has compact values. Hence, $G(t, \omega)$ : $\overline{D(\varphi)} \rightarrow K(\overline{D(\varphi)})$, where $K(\overline{D(\varphi)})$ is the set of all nonempty compact subsets of $\overline{D(\varphi)}$. Theorem 8 implies that $G$ generates a MRDS.

Further we shall remind the next regularity result for solutions of the inclusion

$$
\left\{\begin{array}{c}
\frac{d v}{d t} \in-\partial \varphi(v)+f(t), t \in[0, T], \\
v(0)=v_{0} \in \overline{D(\varphi)} .
\end{array}\right.
$$

Proposition 13 (Brezis [8, p.82] and Barbu [7, p.189]) For any $f(\cdot) \in L_{2}([0, T], X)$, $v_{0} \in \overline{D(\varphi)}$, there exists a unique strong solution of inclusion (19) such that

$$
\begin{gathered}
v(\cdot) \in C([0, T], X), \sqrt{t} \frac{d v}{d t} \in L_{2}([0, T], X), \\
\varphi(v(\cdot)) \in C((0, T]), \varphi(v(\cdot)) \in L_{1}([0, T]),
\end{gathered}
$$

and $\varphi(v(t))$ is absolutely continuous on $[\delta, T], \forall \delta>0$. Moreover,

$$
\left|\frac{d v}{d t}\right|^{2}+\frac{d}{d t} \varphi(v(t))=\left\langle f, \frac{d v}{d t}\right\rangle \text {, a.e. on }(0, T) .
$$

If $u_{0} \in D(\varphi)$ then $\frac{d u}{d t} \in L_{2}([0, T], X)$ and $\varphi(u)$ is absolutely continuous on $[0, T]$.

We note the next important consequence of the preceding proposition. Let us take an arbitrary integral solution of inclusion $(4), v(\cdot) \in \mathcal{D}\left(v_{0}, \omega\right), v(\cdot)=I\left(v_{0}\right) f(\cdot)$. It follows from Lemma 10 that $f(\cdot) \in L_{2}([0, T], X)$. Then, since the solution of (19) is unique for any $v_{0} \in \overline{D(\varphi)}$, it follows that $v(\cdot)$ is a strong solution of (19).

Further, Proposition 13 and Lemma 10 allow us to prove an important property of the map $G$.

Theorem 14 Let property $(H)$ hold. Then for any bounded $B \subset X$, any $T>0$ and $\omega \in \Omega$, there exists $R(\omega)>0$ such that $G(T, \omega) B \subset M_{R(\omega)}$.

Proof. In view of Lemma 10 , the set $M(B, \omega, T)$ is bounded in $L_{\infty}([0, T], X)$ and then it is bounded in $L_{2}([0, T], X)$ for any bounded $B \subset X, T>0, \omega \in \Omega$. We take and arbitrary $v(\cdot) \in \mathcal{D}(B, \omega), v(\cdot)=I\left(v_{0}\right) f(\cdot), v_{0} \in B$. Consider first that $v_{0} \in D(\varphi)$. It follows from equality (20) that

$$
t\left|\frac{d v}{d t}\right|^{2}+t \frac{d}{d t} \varphi(v)=t\left\langle f, \frac{d v}{d t}\right\rangle \text {, a.e. on }(0, T) .
$$


Hence,

$$
\int_{0}^{T} t\left|\frac{d v}{d t}\right|^{2} d t+T \varphi(v(T))=\int_{0}^{T} t\left\langle f, \frac{d v}{d t}\right\rangle d t+\int_{0}^{T} \varphi(v(t)) d t
$$

and then,

$$
\begin{gathered}
T \varphi(v(T)) \leq \frac{1}{2} \int_{0}^{T} t\left|\frac{d v}{d t}\right|^{2} d t+T \varphi(v(T)) \leq \\
\leq \frac{1}{2} \int_{0}^{T} t\|f(t)\|^{2} d t+\int_{0}^{T} \varphi(v(t)) d t .
\end{gathered}
$$

On the other hand, there is no loss of generality in assuming that $\min \{\varphi(v): v \in X\}=$ $\varphi\left(x_{0}\right)=0$. Indeed, let $x_{0} \in D(\partial \varphi), v_{0} \in \partial \varphi\left(x_{0}\right)$. If we introduce the new function $\widetilde{\varphi}(v)=\varphi(v)-\varphi\left(x_{0}\right)-\left(y_{0}, v-x_{0}\right)$, then the equation

$$
\frac{d v}{d t}+\partial \varphi(v) \ni f(t)
$$

is equivalent to

$$
\frac{d v}{d t}+\partial \widetilde{\varphi}(v) \ni f(t)-y_{0}=\widetilde{f}(t)
$$

and $\min \{\widetilde{\varphi}(v): v \in X\}=\widetilde{\varphi}\left(x_{0}\right)=0$. It is clear that $\widetilde{\varphi}$ satisfies also property $(H)$.

Hence, since $f(t)-\frac{d v(t)}{d t} \in \partial \varphi(v(t))$ a.e. on $(0, T)$, we have

$$
\varphi(v(t)) \leq\left\langle f(t)-\frac{d v(t)}{d t}, v(t)-x_{0}\right\rangle
$$

Integrating over $(0, T)$ we get

$$
\begin{aligned}
\int_{0}^{T} \varphi(v(t)) d t \leq & \frac{1}{2}\left\|v(0)-x_{0}\right\|^{2}-\frac{1}{2}\left\|v(T)-x_{0}\right\|^{2}+\int_{0}^{T}\|f(t)\|\left\|v(t)-x_{0}\right\| d t \leq \\
& \leq \frac{1}{2}\left\|v(0)-x_{0}\right\|^{2}+\int_{0}^{T}\|f(t)\|\left\|v(t)-x_{0}\right\| d t .
\end{aligned}
$$

Since $0 \in-\partial \varphi\left(x_{0}\right)$, it follows from inequality (7) that

$$
\left\|v(t)-x_{0}\right\| \leq\left\|v(0)-x_{0}\right\|+\int_{0}^{T}\|f(\tau)\| d \tau, 0 \leq t \leq T .
$$

It follows from the last two inequalities and Lemma 10 that

$$
\int_{0}^{T} \varphi(v(t)) d t \leq\left(\left\|v(0)-x_{0}\right\|+\int_{0}^{T}\|f(t)\| d t\right)^{2} \leq D(\omega)<\infty .
$$

Since the set $M(B, \omega, T)$ is bounded in $L_{\infty}([0, T], X)$ for any bounded set $B, D(\omega)$ does not depend on $v(\cdot) \in \mathcal{D}(B, \omega)$. Using (22) in relation (21) we obtain that, for any $T>0$, there exists $K(\omega)>0$ such that $\varphi(v(T)) \leq K(\omega)$. 
Now let $v_{0} \in B$ be arbitrary. We can assume without loss of generality that $B$ is open and then there exists a sequence $v_{0}^{n} \rightarrow v_{0}$, where $v_{0}^{n} \in D(\varphi), v_{0}^{n} \in B$. In view of (8), for each $v_{0}^{n}$ there exists an integral solution of $(4), v^{n}(\cdot)$ such that

$$
\left\|v^{n}(t)-v(t)\right\| \leq\left\|v_{0}^{n}-v_{0}\right\| \exp (2 C t), \forall t \in[0, T]
$$

Since $\varphi\left(v^{n}(T)\right) \leq K(\omega), \forall n$, and $\varphi$ is lower semicontinuous, we get

$$
\varphi(v(T)) \leq \liminf _{n \rightarrow \infty} \varphi\left(v^{n}(T)\right) \leq K(\omega)
$$

On the other hand, by Lemma $10, \mathcal{D}(B, \omega)$ is bounded in $C([0, T], X)$. Hence,

$$
\|v(T)\| \leq L(\omega)<\infty, \forall v(\cdot) \in \mathcal{D}(B, \omega)
$$

Therefore,

$$
G(T, \omega) B \subset M_{R(\omega)}
$$

where $R(\omega)=\max \{K(\omega)+\|\zeta(T)\|, L(\omega)+\|\zeta(T)\|\}$. It follows from $(H)$ that $G(T, \omega) B$ is precompact in $X$.

Corollary 15 Let property $(H)$ hold. Then, for any $T>0$ and $\omega \in \Omega, G(T, \omega)$ is compact.

Proof. We must prove that for every bounded set $B$, any $T>0$ and $\omega \in \Omega$, the set $G(T, \omega) B$ is precompact in $X$. But this fact follows immediately from $(H)$ and the previous Theorem.

Theorem 16 Let $(F 1)-(F 2),(H)$ and (14) be satisfied. Then, G has the minimal global invariant random attractor $\mathcal{A}(\omega)$, which is measurable with respect to $\mathcal{F}$.

Proof. It is a consequence of Theorem 12 and Corollary 15.

\subsection{Reaction-diffusion inclusions}

Let $f: \mathbb{R} \rightarrow C_{v}(\mathbb{R})$ be a multivalued map. Assume that $f$ is Lipschitz, i.e. $\exists C \geq 0$ such that $\forall x, z \in \mathbb{R}$

$$
\operatorname{dist}_{H}(f(x), f(z)) \leq C\|x-z\| .
$$

Let $\mathcal{O} \subset \mathbb{R}^{n}$ be an open bounded subset with smooth boundary $\partial \mathcal{O}$. Consider the stochastic inclusion

$$
\left\{\begin{array}{c}
\frac{\partial u}{\partial t} \in \Delta u+f(u)+h+\sum_{i=1}^{m} \phi_{i} \frac{d w_{i}(t)}{d t}, \text { on } \mathcal{O} \times(0, T), \\
u=0, \text { on } \partial \mathcal{O} \times(0, T), \\
u(x, 0)=u_{0}(x) \text { on } \mathcal{O},
\end{array}\right.
$$


where $h(\cdot) \in L_{2}(\mathcal{O})$. Define the operators $A: D(A) \rightarrow X, F: X \rightarrow 2^{X}, X=L_{2}(\mathcal{O})$,

$$
\begin{gathered}
A u=\Delta u, \\
F(u)=\{y \in X: y(x) \in f(u(x))+h(x)\},
\end{gathered}
$$

with $D(A)=H^{2}(\mathcal{O}) \cap H_{0}^{1}(\mathcal{O})$. It is assumed that $\phi_{i} \in D(A)$. The map $-A$ is the subdifferential of a proper, convex, lower semicontinuous function $\varphi$ and the map $F$ satisfies $(F 1)-(F 2)$. Moreover, condition $(H)$ is satisfied and $\overline{D(\varphi)}=X$ (see Melnik and Valero [19], Section 3.2.2.). Hence, (24) is a particular case of (18).

We shall assume that there exist $M \geq 0, \delta>0$ such that $\forall s \in \mathbb{R}, \forall z \in f(s)$,

$$
z s \leq\left(\lambda_{1}-2 \delta\right)|s|^{2}+M_{1}
$$

where $\lambda_{1}$ is the first eigenvalue of $-\Delta$ in $H_{0}^{1}(\Omega)$.

We obtain the following theorem:

Theorem 17 Let (25) hold. Then, the MRDS generated by (24) has the minimal global invariant random attractor $\mathcal{A}(\omega)$, which is measurable with respect to $\mathcal{F}$.

Proof. We have to check that (14) holds. In our case $\varepsilon=\lambda_{1}$. In view of (25) for any $y \in D(A)=H^{2}(\mathcal{O}) \cap H_{0}^{1}(\mathcal{O}), \xi \in F(y)$,

$$
\begin{gathered}
(\xi, y) \leq\left(\lambda_{1}-2 \delta\right)\|y\|^{2}+M_{1} \mu(\mathcal{O})+\langle h, y\rangle \\
\leq\left(\lambda_{1}-\delta\right)\|y\|^{2}+M
\end{gathered}
$$

for some $M \geq 0$, so that (14) holds. The statement follows from Theorem 16 .

\section{Conclusions and final remarks}

We have introduced the concept of MRDS by showing the cocycle property and the measurability of the stochastic flows associated to some stochastic differential inclusions with additive noise. Some other examples, also for the existence of random attractors, as linear multiplicative white noise, could have been written. However, this is the subject of a subsequent work. A generalization of the theory of random attractors to this kind of equations has been established, adding some light to a better understanding on the long time behaviour of some stochastic multivalued partial differential equations.

One of the most interesting properties of global attractors for infinite dimensional dynamical systems is that of being compact sets with finite fractal or Hausdorff dimension, as this implies some results on the dependence of the asymptotic behaviour of the

systems with only a finite number of degrees of freedom (cf. Robinson [20]). However, 
for deterministic multivalued partial differential equations is difficult to prove this finite dimensionality of attractors, and we can also find counterexamples where this is not true (cf. Valero [25]). Thus, it would be very interesting to study the dimension of random attractors for MRDS and the relation with the dependence on a finite number of parameters of the long time behaviour of these systems.

\section{Acknowledgements}

The authors would like to thank the referees for many helpful and valuable suggestions which allowed to improve the paper.

This work was begun while the third author was visiting the University of Sevilla. He wish to thank Tomás Caraballo and José A. Langa for their kind hospitality.

\section{References}

[1] AHMED N.U., Nonlinear stochastic differential inclusions on Banach space, Stoch. Anal. Appl. 12 (1994), 1-10.

[2] ARNOLD L., Random Dynamical Systems, Springer Monographs in Mathematics (1998).

[3] AUBIN J.P., CELLINA A., Differential Inclusions, Springer-Verlag, Berlin (1984).

[4] AUBIN J.P., FRANKOWSKA H., Set-Valued Analysis, Birkhäuser, Boston (1990).

[5] BABIN A.V., VISHIK M.I., Attractors of partial differential equations and estimate of their dimension, Russian Math. Surveys 38 (1983), 151-213.

[6] BALL J.M., Continuity properties and global attractors of generalized semiflows and the Navier-Stokes equations, J. Nonlinear Sci. 7 (1997), 475-502.

[7] BARBU V., Nonlinear Semigroups and Differential Equations in Banach Spaces, Editura Academiei, Bucuresti (1976).

[8] BREZIS H., Problemes unilateraux, J. Math. Pures Appl. 51 (1972), 1-168.

[9] CARABALlO T., LANGA J.A., ROBINSON J., Upper semicontinuity of attractors for small random perturbations of dynamical systems, Comm. Partial Differential Equations 23 (1998), 1557-1581.

[10] CASTAING C., VALADIER M., Convex Analysis and Measurable Multifunctions, LNM Vol. 580, Springer (1977).

[11] CRAUEL H., FLANDOLI F., Attractors for random dynamical systems, Prob. Theory Related Fields 100 (1994), 365-393. 
[12] CRAUEL H., Random Probability Measures on Polish Spaces, Habilitationsschrift, Bremen (1995).

[13] CRAUEL H., Global random attractors are uniquely determined by attracting deterministic compact sets, Ann. Mat. Pura Appl. Ser. IV, Vol CLXXVI (1999), 57-72.

[14] DA PRATO G., FRANKOWSKA H., A stochastic Filippov theorem, Stoch. Anal. Appl. 12 (1994), no. 4, 409-426.

[15] FLANDOLI, F., SCHMALFUSS, B.: Random attractors for the 3-D stochastic Navier-Stokes equation with multiplicative white noise, Stochastics Stochastics Rep., 59 (1996), 21-45.

[16] HARAUX A., Attractors of asymptotically compact processes and applications to nonlinear partial differential equations, Comm. Partial Differential Equations 13, 11 (1988), 1383-1414.

[17] HALE J., Asymptotic Behavior of Dissipative Systems, Math. Surveys and Monographs, AMS, Providence (1988).

[18] LADYZHENSKAYA O., Attractors for Semigroups and Evolution Equations, Accademia Nazionale dei Lincei, Cambridge University Press, Cambridge (1991).

[19] MELNIK V., VALERO J., On Attractors of multivalued semi-flows and differential inclusions, Set-Valued Anal. 6 (1998), 83-111.

[20] ROBINSON J.C., Global attractors: topology and finite-dimensional dynamics, $J$. Dynamics Differential Equations 11 (1999) 557-581.

[21] SCHENK-HOPPÉ, K.R.: Random attractors: general properties, existence and applications to stochastic bifurcation theory, Discrete Contin. Dynam. Systems, 4, 1 (1998), 99-130.

[22] SCHMALFUSS B., Backward cocycle and attractors of stochastic differential equations, in V. Reitmann, T. Redrich and N. JKosch (eds.), International Seminar on Applied Mathematics-Nonlinear Dynamics: Attractor Approximation and Global Behaviour (1992), 185-192.

[23] TEMAM R., Infinite Dimensional Dynamical Systems in Mechanics and Physics, Springer-Verlag, New York (1988).

[24] TOLSTONOGOV A.A., On solutions of evolution inclusions.I, Sibirsk. Mat. Zh. 33, 3 (1992), 161-174 (English translation in Siberian Math. J., 33, 3 (1992)).

[25] VALERO J., Finite and infinite-dimensional attractors of multivalued reactiondiffusion equations, Acta Math. Hungar., 88, 4 (2000). 